\title{
The acceptability and feasibility of a planned pre-pregnancy weight loss intervention (the Plan-it Study): A Protocol Paper
}

Elinor Coulman ${ }^{1 *}$, Rebecca Cannings-Johnn ${ }^{1}$, Zoe Couzens ${ }^{5}$, Freya Davies ${ }^{2}$,Josie Henley ${ }^{1}$, Mandy Lau $^{1}$, Fiona Lugg-Widger $^{1}$, Leah Morantz $^{6}$, Julia Sanders ${ }^{3}$, Caroline Scherf ${ }^{4}$, Heather Strange ${ }^{1}$ and Susan Channon ${ }^{1}$

${ }^{1}$ Centre for Trials Research, Cardiff University, UK

${ }^{2}$ The Welsh Centre for Primary and Emergency Care Research (PRIME), Cardiff University, UK

${ }^{3}$ Healthcare Sciences, Cardiff University, UK

${ }^{4}$ Cardiff and Vale University Health Board, UK

${ }^{5}$ Public Health Wales NHS Trust, UK

${ }^{6}$ Patient and Public representative, UK

\begin{abstract}
Background: Women with obesity are at a greater risk of experiencing complications during the antenatal, intrapartum and postpartum periods. Despite many studies of weight management interventions in pregnancy, systematic reviews have demonstrated limited effectiveness, demonstrating a need to increase the focus on preconception health and build a greater awareness of the association between preconception health and maternal and child outcomes. Much of the research in pre-conception weight loss has been with very specific populations or has been small-scale. The aim of the Plan-it study is to establish if it is acceptable and feasible to conduct a study that asks women with overweight/ obesity (BMI of 25 or over) to delay removal of LARC to participate in a targeted pre-pregnancy weight loss intervention.
\end{abstract}

Methods: The study will take a concurrent mixed methods approach incorporating use of routine NHS data and qualitative data collection and analysis across two work-packages: the first will establish the feasibility of defining and understanding the population through routine data and the second will provide an understanding of the feasibility and acceptability to stakeholders (LARC users and practitioners) of incorporating a pre-pregnancy weight loss intervention, in addition to identifying potentially suitable weight loss interventions and the theories underpinning them. The findings from the two work packages will contribute to a final report, which will delineate the key design elements of a future trial or identify the barriers that currently prevent such a trial taking place.

Discussion: The preconception period provides an opportunity to intervene with a weight loss intervention in women with overweight/ obesity. The findings of this study will contribute to the evidence base for weight management in the preconception period and provides information, critical to consider, when developing a future intervention and associated feasibility trial.

Trial registration: ISRCTN14733020

List of abbreviations: BMI: Body Mass Index, CPRD: Clinical Practice Research Datalink. GDPR: General Data Protection Regulation, ISAC: Independent Scientific Advisory Committee, LARC: Long-Acting Reversible Contraceptive, PHW: Public Health Wales, PIS: Participant Information Sheet, SAG: Stakeholder Advisory Group, SHC: Sexual Health Clinics, SMG: Study Management Group, SSC: Study Steering Committee, WP: Work package.

\section{Background}

Women with obesity (those with a body mass index (BMI) of 30 or over) are at a greater risk of experiencing complications during the antenatal, intrapartum and postpartum periods; such complications include gestational diabetes, shoulder dystocia and venous thrombosis. The risk of adverse effects on the child is also increased, such as child obesity [1]. In addition, evidence continues to emerge from longitudinal cohort studies demonstrating future health-related risks such as the increased risk to females born to mothers with overweight or obesity of having polycystic ovary syndrome later in life [2]. Despite many studies of weight management interventions in pregnancy, systematic reviews have demonstrated limited effectiveness; interventions in the antenatal period are associated with modest improvement of physical activity [3], improvements in diet and limited reductions in gestational weight gain, but do not demonstrate improvements in maternal outcomes [4,5].

There is a need to increase the focus on preconception health and build a greater awareness of the association between preconception health and maternal and child outcomes [6]. Much of the research in pre-conception weight loss has been with very specific populations

${ }^{\star}$ Correspondence to: Elinor Coulman, Centre for Trials Research, Cardiff University, UK, E-mail: johne1@cardiff.ac.uk

Key words: preconception, overweight, obesity, intervention, pregnancy, weight management

Received: February 05, 2021; Accepted: February 22, 2021; Published: February 26,2021 
such as gastric by-pass patients [7], couples seeking IVF treatment [8] or has been small-scale [9]. Two Cochrane reviews investigating the effectiveness of preconception and antenatal health programs and interventions to improve pregnancy outcomes or reduce weight in obese women, identified no eligible studies [10,11]. A more recent scoping review of randomised controlled trials of behavioural interventions supporting women of childbearing age in the prevention and treatment of overweight and obesity, only identified one intervention in the preconception period, the Prepare study protocol [12], which has gestational weight gain as the primary outcome.

With the rapidly rising rates of women of childbearing age who are obese, the development of effective pre-pregnancy weight loss interventions for women with overweight/ obesity may provide an important step in reducing health risks to mother and child, but there are challenges to be overcome. As in pregnancy [13], the preconception period may also be considered a "teachable moment" where efforts may be made to positively influence women's diet and health behaviours. However, even though the majority of pregnancies are planned (eg $70 \%$ in a community survey in Australia) [14], women's enhanced motivation to be healthy may not translate into action due to perceived barriers such as time, relevance and care-provider attitudes [9]. Practitioners also experience barriers to raising weight management in pregnancyrelated consultations including lack of skills, time, sensitivity of topic and confidence in the available interventions $[15,16]$. The "preconception" population is largely invisible in health-service terms, with no clear time point of intervention apart from within the small proportion of women who attend surgeries for preconception advice.

Women who use long-acting reversible contraception (LARCs) and who require removal of the device to become pregnant represent a unique group where there is an opportunity for intervention. However, at this point in their reproductive decision-making, it may be difficult to ask women to delay conception through continued use of LARC and engage in weight loss programmes, raising pragmatic and ethical issues for both an intervention and any research study designed to establish effectiveness. A small feasibility study of an intensive weight management programme offered to women with a BMI of 30 or more attending for LARC removal [17] demonstrated that some women were willing to consider delaying LARC removal for six months in order to participate. This small evidence base suggests that there may be an interest in weight loss and a willingness to delay LARC removal in relevant populations. However, with high rates of non-participation and attrition from the programme, it has not yet been established what, if anything, the nature of an acceptable intervention would be.

In order to answer this question, a mixed methods approach is required incorporating use of routine data, qualitative data collection and analysis, and a central role for stakeholders. This will lead to a better understanding of the LARC pathway from an individual and population perspective and its interface with weight management. LARC users' and practitioners' experiences of LARC services, decision making and management of weight around pregnancy will be explored alongside their views of the ethical and methodological issues associated with the timing of informed consent and a potential preconception weight loss intervention. Practitioners' LARC practice and consultation patterns regarding LARC use and removal will be identified utilising datasets, collected routinely across the four UK nations, to compare the population across the different health care settings, as well as over time, taking into account factors such as the impact of different GP incentives on activity and recording $[18,19]$. All this information will be critical to consider when developing a future intervention.

\section{Methods/ Design}

\section{Objectives/ aims}

The aim of the Plan-it study is to establish if it is acceptable and feasible to conduct a study that asks women with overweight/ obesity (BMI of 25 or over) to delay removal of LARC to participate in a targeted pre-pregnancy weight loss intervention.

The study objectives are to identify:

1. The annual number of women of reproductive age (16-48 years old) in the UK who request LARC removal and subsequently have a pregnancy.

2. Means of identifying women with overweight/ obesity at study sites who plan to have LARC removal for the purpose of planning a pregnancy and identify opportunities to intervene.

3. Suitable and acceptable interventions that can be incorporated into a pre-pregnancy weight loss intervention.

4. Willingness of clinicians to raise weight loss in consultations and recruit eligible women to the intervention.

5. Women's views as to the acceptability and feasibility of the proposed intervention.

6. Future potential intervention based on feasibility and acceptability to stakeholders.

\section{Study design}

The study will take a concurrent mixed methods approach incorporating use of routine NHS data and qualitative data collection and analysis across two work-packages (WPs): WP1 will address objectives 1 and 2 and will establish the feasibility of defining and understanding the population through routine data; WP2 will address objectives 3,4 and 5 and will provide an understanding of the feasibility and acceptability to stakeholders (LARC users and practitioners) of incorporating a pre-pregnancy weight loss intervention, in addition to identifying potentially suitable weight loss interventions and the theories underpinning them. The findings from the two work packages will be brought together in the final report of the study, addressing objective 6 , which will delineate the key design elements of a future trial or, depending on stakeholder response, identify the barriers that currently prevent such a trial taking place.

Work Package 1: Defining and understanding the population through routine data: This WP will use routine data from Welsh Sexual Health Clinics (SHC) and UK General Practices relating to women attending for LARC removal to:

1. understand the pattern of LARC use to identify opportunities to intervene;

2. report the annual number of women in the UK requesting removal of LARC without replacing it with an alternative prescribed contraception;

3. identify women requesting LARC removal who subsequently become pregnant who would be eligible to recruit to a weight loss intervention study;

4. identify events in GP and hospital records to explore time from LARC removal to conception or appointments relating to difficulties conceiving (if possible). 
Work Package 2: Understanding context and stakeholder views: WP2 will be conducted in two phases.

Phase 1 comprises

i) Scoping work to identify suitable weight loss and weight-related health behaviour interventions and the theories that underpin them.

ii) Developing an understanding of typical pre-conception pathways related to LARC/ LARC removal from the perspectives of service users and service providers.

Phase 2 compromises establishing the acceptability and feasibility of proposed intervention and study design.

\section{Work Package 2 Phase 1: Scoping suitable interventions and underlying theories}

Theories regarding how and when health behaviour interventions in the pre-conception phase may function will be developed, guided by the principles of scientific realism [20]. Studies identified in systematic reviews of weight loss interventions prior to and during pregnancy and relevant behaviour change interventions, companion papers (qualitative studies and process evaluations) and grey literature will provide an understanding of how and when preconception weight loss interventions might successfully be applied. Identified health gains or risks to health associated with the intervention will be incorporated into the review, paying particular attention to factors which potentially could improve or compromise health in the context of preparation for conception. The scope of the review will be limited to systematic reviews and relevant papers published in order to use the literature to build and develop early theories for later refinement, rather than to conduct a full realist synthesis which might also aim to test these theories. Barriers and facilitators to engagement in pre-conception health behaviour change interventions will be considered and will focus theory development. The outcome of this task will be a set of contextmechanism-outcome configurations and key components of the intervention which will be taken forward to our Stakeholder Advisory Groups as the basis of an early overall programme theory, to be further developed within Phase 2.

\section{Work Package 2 Phase 1: Understanding the preconception pathways relating to $\mathrm{LARC}$}

A range of qualitative methods will be used to generate a detailed understanding of typical pre-conception pathways related to LARC use/ LARC removal from the perspectives of both service users and service providers, including: the LARC removal service contexts, how LARC are managed by women and their clinicians, the interrelationship between discussions about weight/ obesity and family planning, feasible opportunities to intervene, potential intervention components including additional health-related content (e.g. folic acid, availability of smoking cessation services). This phase will comprise of 3 components: 1) Analysis of policy documents, 2) Engagement with LARC Users and 3) Professional engagement (practitioners and weight loss consultants).

\section{Analysis of policy documents}

UK national policies and guidelines and a number of local policies (based on availability and saturation of core concepts) will be reviewed to understand how services are expected to approach discussions of weight loss with women with overweight/ obesity, how LARC treatment pathways currently operate, guidance on health behaviours prior to conception and the practical/ ethical challenges to successful service delivery in current service structures including equity of access to interventions.

\section{Engagement with LARC Users}

Qualitative surveys using open-text questions [21] will be utilised to understand women's knowledge of the risks of overweight/ obesity in pregnancy, women's experiences of discussing weight with healthcare professionals, how, where and when women currently access prepregnancy weight loss interventions, barriers and facilitators to the introduction of a weight loss intervention at a LARC removal appointment and preferable components of a potential intervention.

An online qualitative survey of women of reproductive age who self-identify as having/ previously having overweight/ obesity will be advertised through a range of relevant social media platforms. A minimum of 200 and a maximum of 500 responses will be collected. Data collection will cease when sufficient 'information power' has been generated [22].

\section{Professional engagement (Practitioners and weight loss consultants)}

Practitioners (GPs, nurses) who insert or remove coils as part of their role will be identified and recruited at up to eight relevant professional meetings. Qualitative surveys with practitioners using open-text questions [21] will focus on any pre-pregnancy care provision, the discussion of weight and pre-conception health both generally and in the specific context of LARC removal, challenges to service delivery, equity of access to interventions and their views on the potential for an intervention postponing LARC removal as part of pre-conception weight loss plan. Weight loss consultants (e.g. people who support women to lose weight as part of their role) will be recruited via existing contacts or a range of social media platforms. Qualitative surveys will address questions of feasibility and their experiences/ views on the provision of weight loss programmes in the pre-conception phase.

\section{Stakeholder advisory groups}

At the end of Phase 1 the information gathered will be synthesised to describe the core components of a potential intervention, together with the contextual factors likely to be important influences on outcomes and study designs. This will be refined through work with two Stakeholder Advisory Groups (SAGs); a LARC User and a practitioner SAG will comprise up to 10 LARC Users or practitioners respectively and all will be recruited through the exploratory work in Phase 1 . The practitioner SAG will be run as part of a Continuing Professional Development event in order that practitioners can be incentivised to attend but without disruption to clinical activity. The LARC user SAG will take the form of either a focus group (face-to-face or virtually), individual qualitative interviews or electronic group chat portal. Both SAGs will work with the research team to generate potential intervention and study designs which are in keeping with the findings of Phase 1 and consider the issue of equity of access to the intervention. They will also consider the key questions to ask participants in Phase 2. The two SAGs will advise the study team again at the end of Phase 2.

\section{Phase 2: Acceptability and feasibility of proposed intervention \& study design}

The outputs from Phase 1 will be explored in Phase 2 with targeted qualitative work addressing acceptability and feasibility of a potential weight loss intervention and study design to women in the target population and to practitioners. Phase 2 qualitative interview schedules 
will be informed by the feedback from the SAGs and the interviews will further test and refine the theories developed in Phase 1. Up to 20 participant interviews and 10 professional interviews will be conducted face-to-face, virtually or over the telephone. Practitioners who remove LARCs and practitioners delivering community weight loss interventions will be asked to explore their views about the type of intervention, the potential study designs and, for clinicians, their willingness to recruit women to such a study. LARC Users will be asked to explore their views of the acceptability and feasibility of the potential weight loss intervention and study design. Findings from the targeted qualitative Phase 2 work will be discussed with the LARC User and Practitioner SAGs. The SAGs will take the form of a focus group (either face-to-face or virtually). At the end of Phase 2 the final report will describe the key design elements of a potential future study or the reasons why such a trial is currently not feasible to deliver.

\section{Theoretical/ conceptual framework}

WP2 work will be guided by the principles of Pawson and Tilley's scientific realism [20]. This approach was designed for the evaluation of complex social interventions based on realist understanding of causation which recognises that social interventions lead to outcomes by triggering a reaction within individuals. The way in which individuals respond to interventions is understood to relate both to characteristics of the interventions themselves, and also characteristics of the context into which the intervention is delivered. Realist research focusses on the development of explanatory theories which describe how all of these factors interact using context-mechanism-outcome configurations. A range of different sources of data as part of an iterative process of theory development and refinement will be used, recognising that different stakeholders may illuminate different important theories. The resultant programme theory can be used to guide decisions about which interventions are most likely to succeed in which settings. It could also guide a theory driven evaluation of any future feasibility study.

\section{Study processes}

\section{Participant identification/ selection}

WP1 participants will be included in routine datasets if they meet eligibility criteria (Table 1 ).

In WP2, LARC Users will self-refer to adverts in relevant social spaces in online locations, including Facebook and Twitter. An advert will also be distributed via Healthwise Wales (a national registry) [23].
The range of online spaces targeted will be purposively broad in order to maximise involvement across a range of demographic backgrounds. WP2 Phase 1 participants in the practitioner survey will be recruited at up to 8 relevant professional meetings (professional meeting targeted at sexual health clinicians), which will be attended by the study team. Professionals delivering weight loss interventions will be recruited via existing networks of widely used community based weight loss programmes and advertisement through social media platforms. LARC user SAG members and WP2 Phase 2 interviewees will be recruited from a pool of participants who have previously consented to be contacted in the exploratory work in WP2 Phase 1 online surveys. LARC users will be purposively sampled to encourage membership from participants with different BMI values and a range of reproductive histories and future plans. Practitioner SAG members will be recruited at a relevant professional event and WP2 Phase 2 practitioner interviewees will be recruited from a pool of WP2 participants who have previously consented to be contacted in the exploratory work in WP2 Phase 1 online surveys.

\section{Eligibility criteria}

Participants will be included in routine datasets if they meet the eligibility criteria and in the online surveys if they self-identify as meeting the eligibility criteria, as detailed in Table 1.

\section{WP2: Phase 2 participant interviews}

Participants will be recruited from a pool of WP2 participants who have previously consented to be contacted. As all participants will have met eligibility criteria for WP2 Phase 1 online survey inclusion, there will not be specific eligibility criteria for WP2 Phase 2 interviews.

\section{Participant informed consent}

WP1: Routine data: Anonymised data and aggregate/ open data will be accessed and therefore consent is not required [24].

WP2 Phase 1: Online surveys. Participants will be asked to confirm eligibility in the online survey. Participants will be provided information about the study in the form of a Participant Information Sheet (PIS), which they will be required to read, and inclusion and exclusion criteria will be clearly specified in the publicity materials and consent procedure. Participants will be told to take as much time as required to consider the information before taking part in the study. Participants will be screened and electronic consent collected on the online survey.

Table 1. Participant eligibility criteria

\begin{tabular}{|c|c|c|}
\hline & Inclusion criteria & Exclusion criteria \\
\hline \multicolumn{3}{|c|}{ WP1: Routine Data } \\
\hline \multicolumn{3}{|l|}{ Data sources } \\
\hline Clinical Practice Research Data & $\begin{array}{l}\text { Women of reproductive age (16-48 years old) who: } \\
\text { i) have a LARC inserted/ in situ/ removal Read code between } 1^{\text {st } J a n u a r y ~} 2009 \text { and } 31^{\text {st }} \text { December } 2018 .\end{array}$ & None \\
\hline Sexual Health Clinics data & $\begin{array}{l}\text { Women of reproductive age (16-48 years old) who: } \\
\text { ii) have a LARC inserted/ in situ/ removal Read code between } 1^{\text {st } J a n u a r y ~} 2012 \text { and } 31^{\text {st }} \text { December } 2018 .\end{array}$ & None \\
\hline \multicolumn{3}{|c|}{ WP2: Phase 1 online surveys } \\
\hline \multicolumn{3}{|l|}{ Survey participants } \\
\hline LARC Users & $\begin{array}{l}\text { Women of reproductive age (16-48 years old) who: } \\
\text { i) have experience of using LARC. } \\
\text { ii) who think/ know either that their current weight would put them in the overweight/ obese category or their } \\
\text { weight in the past would have put them in the overweight/ obese category. }\end{array}$ & $\begin{array}{l}\text { Sufficient written English to } \\
\text { participate in online surveys and } \\
\text { consent to participate in the study. }\end{array}$ \\
\hline Practitioners & Clinicians who remove LARC as part of their clinical role & \\
\hline Weight loss consultants & Professionals who support women to lose weight as part of their role. & $\begin{array}{l}\text { Sufficient written English to } \\
\text { participate in online surveys and } \\
\text { consent to participate in the study. }\end{array}$ \\
\hline
\end{tabular}


If participants are eligible and have consented electronically, they will be able to complete the online survey. Participants will be asked to provide contact details for future contact. There will be no follow-up assessments.

For Practitioner online surveys only, participants will be recruited at relevant professional events. Study team members will explain the study to participants and will be available to answer any questions, before following the online survey consent procedure or complete the survey on hard copy.

WP2: LARC User SAG. For the LARC User SAGs, a member of the study team will contact the participant by their provided method of contact (email or telephone) explain the study in detail and will provide the participant with the PIS and will give sufficient time to consider the information. If the meeting is face-to-face, the participants will be asked to sign a consent form at the SAG meeting. If the meeting is held virtually, participants will be sent consent statements by email and asked to confirm their consent by email. The consent process will then be repeated verbally at the SAG meeting.

WP2: Practitioner SAG. Practitioner SAG members will be recruited at a relevant professional event. Attending practitioners will be asked to attend a SAG discussion if appropriate to their professional role. A PIS will be provided to all attendees, the study will be explained in detail to all attending professionals.

WP2: Phase 2 Practitioner and LARC User interviews. Practitioners and LARC Users who have previously consented to this will be contacted by their preferred method of contact (email or telephone) and asked to take part in a further qualitative interview. The study will be explained in detail, the participant will be provided with a PIS and will be given sufficient time to consider the information. Participants will be screened and consented face-to-face, virtually or over the telephone. If face-to-face, the participants will be asked to sign a consent form at the interview. If the interview is to be held virtually or over the telephone, participants will be sent consent statements by email and asked to confirm their consent by email. The consent process will then be repeated verbally at the interview. If participants are eligible and have consented, they will be asked to complete a short qualitative interview. There will be no follow-up assessments.

\section{Data collection}

\section{WP1: Routine data providers and datasets}

Clinical practice research datalink: The CPRD provides UKwide individual anonymised patient GP data. Data covers over $20 \%$ of general practices in the UK and is representative of practices by country, rurality and deprivation quintiles [24]. Data requests of anonymised primary care patient data from CPRD can also include data linked to secondary care and other health-based datasets including the Pregnancy Register. The Pregnancy Register is created by an algorithm that lists all pregnancies identified in the CPRD database [25]. For pregnancies resulting in live births, de-identified information of the linked babies in the CPRD Mother Baby Link are also provided (baby ID, baby month and year of birth, pregnancy start and end date, gestation days, and mother age of pregnancy). LARC related Read codes will be identified using the Read code dictionary and from the literature and will be reviewed by the clinical co-investigators for accuracy and inclusivity of all possible codes. Cardiff University hold an Academic Risk Sharing Licence with CPRD and following Independent Scientific Advisory Committee (ISAC) approval and data will be made available via a Cardiff University data analyst.

\section{Sexual health clinic data}

SHCD data is collected and held separately in Wales, England and Scotland. Public Health Wales (PHW) holds individual level patient data from all SHC in Wales (from 2012) and will be made available to the study team in an aggregate format based on data requirements. Aggregated data from PHW will be transferred to Cardiff University servers upon agreement of data release. The required tabulations of outputs will be agreed with PHW to ensure the eligibility applied to CPRD patients is also applied to the SHCD. In Scotland and England, SHCD is collated and reported by NHS National Services Scotland and NHS Digital respectively. National statistics annual reports on sexual health service use provide open data tables that can be used as a national comparison with Wales (with no formal data access requests required for these open data tables) [25].

\section{WP2: Qualitative data collection}

WP2 Phase 1 qualitative online surveys of LARC Users, practitioners and weight loss consultants will be collected on an online survey, hosted by Cardiff Online Survey on secure Cardiff University servers. SAG meetings and WP2 Phase 2 interviews will be audio-recorded on encrypted audio-recorders.

\section{Study flowchart}

Figure 1 illustrates the study flowchart.

\section{Data management and confidentiality}

All procedures for data storage, processing and management will comply with the Centre for Trials Research Standard Operating Procedures, CPRD \& PHW data sharing agreements and the General Data Protection Regulation (GDPR). Data accessed for WP1 will be anonymised and/ or aggregated prior to receipt and therefore there is no risk of re-identification of patients. All electronic data will be stored on fire walled University servers. Access to files will be through password protected PCs and only accessible to named researchers. Data transfer will be secure using the data providers preferred secure transfer method. The Data controllers are CPRD and PHW. Cardiff University will be the Data Processor.

The online survey will be hosted by Cardiff Online Survey (previously Bristol Online Surveys), on a Cardiff University secure server and access password protected. A member of the research team will act as administrator.

Hard copy consent forms will be stored in a locked filing cabinet. All electronic data, including consent/ contact details will be stored in password-protected servers maintained on Cardiff University networks. All electronic identifiable data (consent, contacts forms) will be stored separately from data. Interviews and SAGs will be recorded on encrypted password protected audio-recorders and voice files will remain password protected and only accessible to relevant members of the research team once transferred to secure Cardiff University servers. Recordings will be transcribed and pseudonymised in line with the Centre for Trials Research Standard Operating Procedures. All essential documents generated by the study will be kept in the Study Master File and/ or on the electronic Study Master File. A full Data Management Plan will be developed. No identifiable data will be published. All data will be kept for 15 years in line with Cardiff University's Research Governance Framework Regulations for clinical research. 
WP 1: Defining and understanding the population

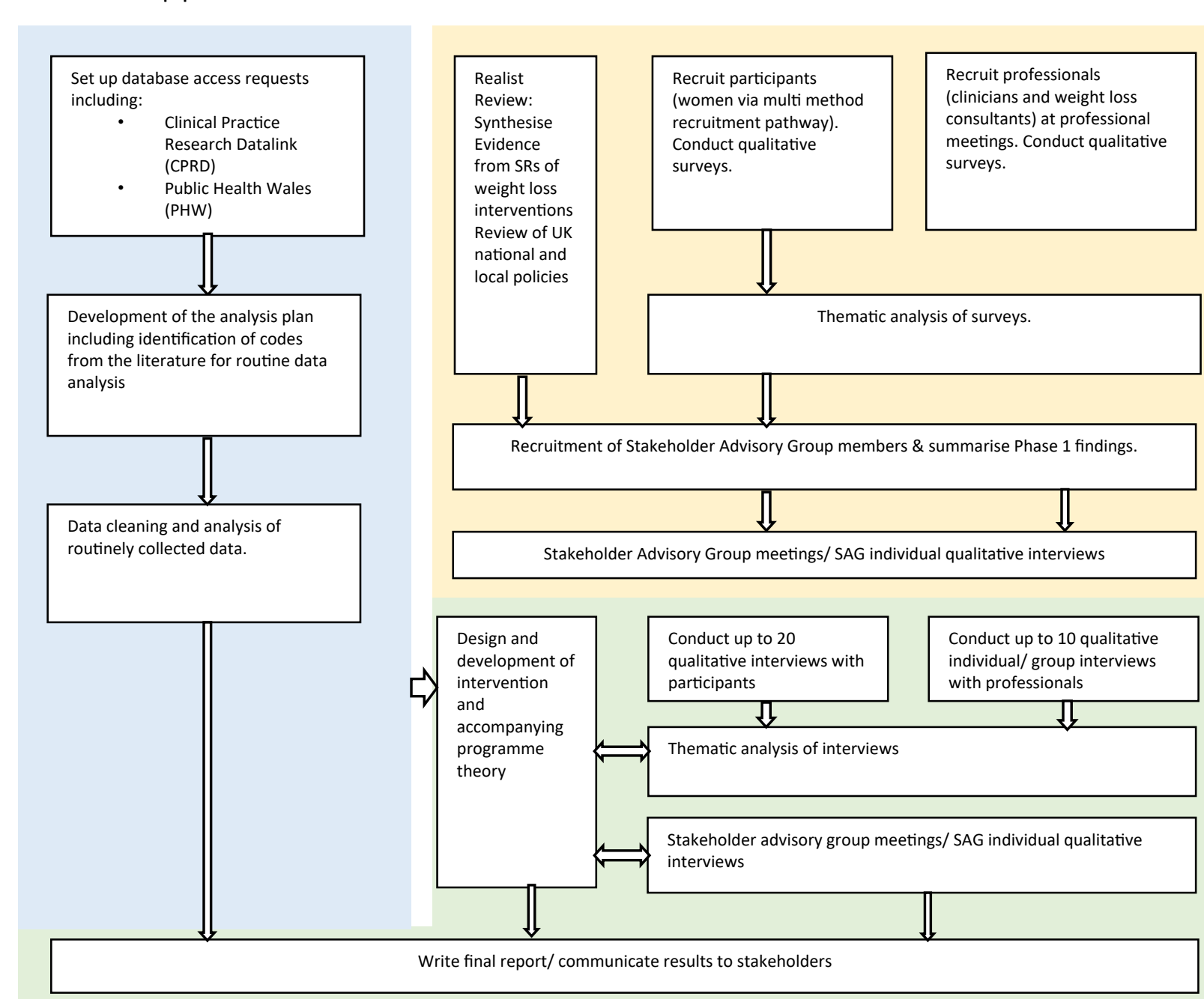

Phase 1

Data

gathering/

theory

development

Figure 1. Plan-it study flow chart

\section{Analysis}

\section{WP1: Routine data}

For the individual anonymised data from CPRD, rates of fitting and removal of LARC in general practice will be calculated and reported over time (either quarterly or annually depending on numbers). Rates are calculated as the number of LARCs fitted and removed as a proportion of all women of reproductive age.

Trends in rates will be examined by country, LARC type, and where available, by attendance type (pre-booked vs walk in consultations). Similarly, we will examine trends using the Welsh SHCD. This will enable a comparison of the case-mix of women recorded for a LARC removal in GP and those who visit a SHC by age, ethnicity, BMI and deprivation. The data from SHCs in Scotland and England will be in aggregate format at NHS Sexual and Reproductive Service provider level and will report rates of fitting and removal annually. Trends in rates by age group at time of fitting/ removal, change of contraception method from/ to LARC and if recorded BMI category and deprivation quintile will be explored. These rates will be compared to those arising from the SHCD in Wales. The quality of recording of BMI in all datasets will be explored. Previous work in the CPRD show that completeness of BMI has increased over time (to around 77\%) and was higher in females, especially in those of reproductive age [27].

Analysing these datasets will identify variation in numbers, pattern and duration of use of LARCs in the different health settings, geographical areas (rural/ urban) and demographic groups. It will be possible to consider what opportunities (e.g., consultation types, frequency of consultations) are available to intervene in the different service delivery designs across the UK. The routine data analysis will determine the most appropriate LARC removal settings, the annual numbers of potential participants available to recruit and an indicative time-frame for recruitment.

For patients attending their GP for fitting or removal of their LARC, the data will allow exploration of the duration of LARC use prior to removal, the changes to contraception use over time and through linking to the Pregnancy Register algorithm, a pregnancy episode related to the women in the cohort (estimated start of pregnancy) will 
be flagged. Accessing these data will enable a broader understanding of the population for whom this intervention will be targeted and potentially identify those who had a LARC removed for the purpose of planning a pregnancy. Examination of how time to conception may differ between BMI and age categories will also be possible. For women where a pregnancy is identified following a LARC removal we will explore the natural distribution of the time lag between these two events to apply a rule to indicate the pregnancy and associated LARC removal was planned.

Whilst the data between GP practices and SHCD cannot be linked, the recording of a LARC removal in a SHC setting will be explored in the GP notes. Previous work using an alternative primary care data source (The Health Improvement Network - THIN) identified that $24 \%$ of LARC related records in primary care came from SHC letters [28].

\section{WP2: Understanding context and stakeholder views}

WP2 online surveys will be analysed using a combination of inductive and deductive thematic analysis. Inductive codes will be generated during analysis and within regular qualitative analysis meetings between the qualitative researchers and the appointed Research Associate. Deductive codes will be developed with reference to a set of key context-mechanism-outcome configurations identified via the realist theory development process. Codes will aim to identify (i) essential elements of the intervention; (ii) contextual factors and (iii) barriers and facilitators to acceptability/ feasibility.

The findings from Phase 1 of WP2 will be synthesized to generate potential model(s) of intervention and study design. The model and intervention-design process will be informed by and draw upon existing formal theories as identified by the review. At the end of Phase 1 the SAGs will meet with the research team to consider the synthesised findings and advise on the intervention and study development. In Phase 2 of WP2, the qualitative data from practitioners and service users will be analysed thematically, using deductive codes focused around the developing programme theory and inductive codes when new information arises.

\section{Withdrawal}

For WP1, data will be aggregated and anonymised, and therefore it will not be possible to remove records once an extract has been produced. For WP2, participants will be able to withdraw at any time by contacting the study team. However, once analysis has taken place, participants will not be able to withdraw data already collected.

\section{Public involvement}

In addition to two Stakeholder Advisory groups, the Study Management Group and Study Steering Committee will include an independent lay representative with experience of overweight/ obesity and pregnancy.

\section{Study management}

The Study Management Group (SMG) will comprise of all team members and co-applicants and will meet approximately bimonthly. A Study Steering Committee (SSC) will meet at least three times throughout the duration of the study to provide study oversight. The SSC comprises of one independent Chair with expertise in diet and lifestyle behaviour change, independent members with experience in realist review methodology, statistical epidemiology and qualitative research and a lay representative.

\section{Discussion}

This study will integrate quantitative data in WP1 and qualitative in WP2 to allow a better understanding of the LARC pathway and its interface with weight management. The use of routine data may provide an opportunity to widely examine practitioners' LARC practice and consultation patterns regarding LARC use and removal across the four UK nations, and provide information regarding the annual numbers of potential participants and the most appropriate LARC removal settings. However, it will not be possible to definitely determine from the routine data if a LARC was removed with the intention to start a family, but by following up patients, it will provide more insight with regards to women's assumed motivation for LARC removal.

The preconception period provides an opportunity to intervene with a weight loss intervention in women with overweight/ obesity. The findings of this study will contribute to the evidence base for weight management in the preconception period and provides information, critical to consider, when developing a future intervention and associated feasibility trial.

\section{Declarations}

\section{Ethics approval and consent to participate}

Ethical approval for this study was given by the Cardiff University School of Medicine Research Ethics Committee on the $30^{\text {th }}$ April 2019, reference number 19/ 42.

\section{Consent for publication}

As this is a protocol paper, this is non-applicable. However, for the study results, a publication plan and dissemination policy will be written. The study results will be disseminated in full and with a lay summary on the Centre for Trials Research (CTR) website and a summary of the results will be disseminated to all participants.

\section{Availability of data and materials}

As this is a protocol paper, this is non-applicable. Any data requests for the study results should be made to the CTR. The CTR is a signatory of AllTrials and aims to make its research data available wherever possible.

\section{Competing interests}

The authors declare that they have no competing interests

\section{Funding}

The Plan-it Study is funded by the National Institute for Health Research, Health Technology Assessment Programme. Ref 17/130/05. The National Institute for Health Research, Health Technology Assessment Programme funded the study under a commissioned call but were not involved in the design of the study or collection, analysis, interpretation of data or writing the manuscript. The Centre for Trials Research receives funding from Health and Care Research Wales and Cancer Research UK.

\section{Authors' contributions}

Study conception: SC. Study protocol: All authors. Drafting manuscript: EC, SC, RCJ, ML and FLW. Study Management: EC. WP1 Lead: FLW. WP2 Lead: HS. Statistical lead: RCJ. All authors critically 
reviewed and approved the final version of the submitted manuscript. SC is the lead investigator.

\section{Acknowledgements}

Not applicable

\section{References}

1. Marchi J, Berg M, Dencker A, Olander EK, Begley C (2015) Risks associated with obesity in pregnancy, for the mother and baby: a systematic review of reviews. Obes Rev 16: 621-638. [Crossref]

2. Valgeirsdottir H, Vanky E, Sundström -Poromaa I, Roos N, Løvvik TS, et al. (2018) Prenatal exposures and birth indices, and subsequent risk of polycystic ovary syndrome: a national registry-based cohort study. BJOG 126: 244-251. [Crossref]

3. Flannery C, Fredrix M, Olander EK, McAuliffe FM, Byrne M, et al. (2019) Effectiveness of physical activity interventions for overweight and obesity during pregnancy: a systematic review of the content of behaviour change interventions. Int $J$ Behav Nutr Phys Act 16: 97. [Crossref]

4. Flynn AC, Dalrymple K, Barr S, Poston L, Goff LM, et al. (2016) Dietary interventions in overweight and obese pregnant women: a systematic review of the content, delivery, and outcomes of randomized controlled trials. Nutr Rev 74: 312-328. [Crossref]

5. Muktabhant B, Lawrie TA, Lumbiganon P, Laopaiboon M (2015) Diet or exercise, or both, for preventing excessive weight gain in pregnancy. Cochrane Database Syst Rev CD007145. [Crossref]

6. Stephenson J, Heslehurst N, Hall J, Schoenaker DAJM, Hutchinson J, et al. (2018) Before the beginning: nutrition and lifestyle in the preconception period and its importance for future health. The Lancet 391: 1830-1841. [Crossref]

7. Adams TD, Hammoud AO, Davidson LE, Laferrère B, Fraser A, et al. (2015) Maternal and neonatal outcomes for pregnancies before and after gastric bypass surgery. Int $J$ Obes (Lond) 39: 686-694. [Crossref]

8. Legro RS, Dodson WC, Kris-Etherton PM, Kunselman AR, Stetteret CM, et al. (2015) Randomized controlled trial of preconception interventions in infertile women with polycystic ovary syndrome. J Clin Endocrinol Metab 100: 4048-4058. [Crossref]

9. Harden SM, Ramalingam NS, Wilson KE, Evans-Hoeker E (2017) Informing the development and uptake of a weight management intervention for preconception: a mixedmethods investigation of patient and provider perceptions. BMC Obes 4: 8. [Crossref]

10. Opray N, Grivell RM, Deussen AR, Dodd JM (2015) Directed preconception health programs and interventions for improving pregnancy outcomes for women who are overweight or obese. Cochrane Database Syst Rev CD010932. [Crossref]

11. Furber CM, McGowan L, Bower P, Kontopantelis E, Quenby S, et al. (2013) Antenatal interventions for reducing weight in obese women for improving pregnancy outcome. Cochrane Database Syst Rev CD00933. [Crossref]

12. LeBlanc ES, Vesco KK, Funk KL, Karanja N, Smith N, et al. (2016) Prepare, a randomized trial to promote and evaluate weight loss among overweight and obese women planning pregnancy: Study design and rationale. Contemp Clin Trials 49: 174180. [Crossref]
13. Phelan S (2010) Pregnancy: a "teachable moment" for weight control and obesity prevention. Am J Obstet Gynecol 202: 135.e1-8.

14. Lang AY, Hall JA, Boyle JA, Barrett G (2019) Validation of the London measure of unplanned pregnancy among pregnant Australian women. PLoS One 14: e0220774. [Crossref]

15. Stotland N, Gilbert P, Bogetz A, Harper C, Abrams B, et al. (2010) Preventing excessive weight gain in pregnancy: how do prenatal care providers approach counseling? $J$ Womens Health (Larchmt) 19: 807-814. [Crossref]

16. Blackburn M, Stathi A, Keogh E, Eccleston C (2015) Raising the topic of weight in general practice: perspectives of GPs and primary care nurses. BMJ Open 5: e008546. [Crossref]

17. Brackenridge L, Finer N, Batterham RL, Pedram K, Ding T, et al. (2018) Prepregnancy weight loss in women with obesity requesting removal of their intra-uterine contraceptive device in order to conceive: a pilot study of full meal replacement. Clin Obes 8: 244-249. [Crossref]

18. Arrowsmith ME, Majeed A, Lee JT, Saxena S (2014) Impact of pay for performance on prescribing of long-acting reversible contraception in primary care: An interrupted time series study. PLoS One 9: e92205. [Crossref]

19. Quality and Outcomes Framework. Available from: http://www.nhsemployers.org/-/ media/Employers/Documents/Primary-care-contracts/QOF/2017-18/201718-Qualityandoutcomes-framework-summary-of-changes.pdf

20. Pawson R, Tilley N (1997) Realistic evaluation. London: Sage.

21. Clarke V, Braun V (2013) Successful qualitative research: a practical guide for beginners. London: Sage.

22. Malterud K, Siersma V, Guassora A (2015) Sample size in qualitative interview studies: Guided by information power. Qual Health Res 26:1753-1760. [Crossref]

23. Healthwise Wales. Available from: www.healthwisewales.gov.wales. [Accessed 14th March 2018]

24. Herrett E, Gallagher AM, Bhaskaran K, Forbes H, Mathur R, et al. (2015) Data resource profile: clinical practice research datalink (CPRD). Int J Epidemiol 44: 827836. [Crossref]

25. Minassian C, Williams R, Meeraus WH, Smeeth L, Campbell OMR, et al. (2019) Methods to generate and validate a Pregnancy Register in the UK Clinical Practice Research Datalink primary care database. Pharmacoepidemiol Drug Saf 28: 923-933. [Crossref]

26. Sexual and Reproductive Health Services (Contraception), England, 2018/19 [National Statistics]. Available at: https://digital.nhs.uk/data-and-information/publications/ statistical/sexual-and-reproductive-health-services (Accessed July 2020).

27. Bhaskaran K, Forbes HJ, Douglas I, Leon DA, Smeeth L (2013) Representativeness and optimal use of body mass index (BMI) in the UK Clinical Practice Research Datalink (CPRD). BMJ Open 3: e003389. [Crossref]

28. Soriano LC, Wallander M, Andersson S, Filonenko A, Rodriguez LAG (2015) The continuation rates of long-acting reversible contraceptives in UK general practice using data from The Health Improvement Network. Pharmacoepidemiol Drug Saf 24: 52-58. [Crossref]

Copyright: (C2021 Coulman E. This is an open-access article distributed under the terms of the Creative Commons Attribution License, which permits unrestricted use, distribution, and reproduction in any medium, provided the original author and source are credited. 